\title{
Effects of a Few Minutes of Abdominal Drawing-in Maneuver on Balance Test Results
}

\author{
Shahram Mohaghegh ${ }^{1, *}$ and Maryam Hajian ${ }^{2}$ \\ ${ }_{1}^{1}$ Research Center For Health Management in Mass Gathering, Red Crescent Society of the Islamic Republic of Iran, Tehran, Iran \\ 2 Community Medicine Specialists, Research Center for Addiction and Risky Behaviors, Iran University of Medical Sciences, Tehran, Iran
}

* Corresponding author: Shahram Mohaghegh, Sports and Exercise Medicine Specialist, Iran-Helal Institute of Applied-Science and Technology, Red Crescent Society of the Islamic Republic of Iran, Tehran, Iran. Tel: +9891230409654; Email: shahrammohaghegh5@gmail.com

Received 2021 February 03; Revised 2021 February 28; Accepted 2021 April 21.

\begin{abstract}
Background: The abdominal draw-in maneuver (ADIM) has been suggested to enhance core stability through the improvement of lumbopelvic stability. Although the effect of ADIM and core muscle activation on some biomechanical parameters has been studied, the effect of exercising a few minutes of this maneuver on balance test results has not been established yet.

Objectives: This study aimed to determine the effect of exercising a few minutes of ADIM on balance test results.

Methods: This study included 18 apparently healthy participants in the age range of 18-60 years. The participants performed a fall risk test using the Biodex balance system. Afterward, the participants were instructed to perform the abdominal drawing-in maneuver. The fall risk test was repeated while the participants were performing this maneuver. The results were compared with the data obtained from participants at the beginning of the study using paired t-test. Data were analyzed according to the participants' BMI (more or less than 25 $\mathrm{kg} / \mathrm{m}^{2}$ ) or abdominal circumference (more or less than $100 \mathrm{~cm}$ ) to reveal the effect of doing ADIM on balance scores in these subgroups.

Results: Based on the obtained results, a significant improvement was observed in the means of balance scores after the participants performed the ADIM. Further analysis revealed a significant improvement in the means of balance scores of individuals with abdominal circumference more than $100 \mathrm{~cm}$ or BMI more than $25 \mathrm{~kg} / \mathrm{m}^{2}(\mathrm{P}<0.05)$.

Conclusion: The short-term impairment of postural control can be dangerous, especially for elderly individuals with abdominal obesity in risky situations, such as mass gatherings. Therefore, it is recommended that individuals with a high risk of falling should be instructed to practice ADIM correctly.

Keywords: Abdominal obesity, Abdominal draw-in maneuver, Balance, Fall, Mass gathering
\end{abstract}

\section{Background}

Postural balance is the capacity to obtain equilibrium by maintaining the body's center of gravity (CoG) over the body's base of support (1). Maintenance of postural balance includes the perception of sensory inputs from body motions, processing of sensorimotor information in the Central Nervous System (CNS), and implementation of suitable musculoskeletal responses. It depends on rapid, continuous processing of visual, vestibular, and somatosensory inputs followed by smooth and synchronized neuromuscular actions (2).

Balance training has been used as part of the preventive programs for ankle and knee injuries during sports $(3,4)$. The weak core musculature is a major cause of incompetent movements that can lead to tissue damage $(5,6)$. The transverse abdominal ( TrA) muscle, is considered an important part of the core muscles (7). It is believed that the abdominal muscles and the deep spine muscles (i.e. TrA and multifidus) are automatically activated prior to the limb muscles in functional upper and lower limb movements, such as arm lifting or walking (8). It has been suggested that the abdominal draw-in maneuver (ADIM) can enhance core stability by the improvement of lumbopelvic stability $(9,10)$. This maneuver is used to activate the TrA muscle as one of the motor control exercises for lumbar stabilization training programs. The ADIM is considered the basic exercise for spinal stabilization and restores proper neuromuscular control and strengthens the muscles of the lateral wall of the abdomen, including TrA, Internal Oblique (IO), and External Oblique (EO) $(11,12)$.

Acute TrA contraction can increase the tension of the thoracolumbar fascia and intra-abdominal pressure (13). The thoracolumbar fascia attaches the lower limbs (via the gluteus maximus) to the upper limbs (via the latissimus dorsi) and allows the core to be involved in coherent kinetic chain activities. It covers deep muscles of the back and trunk including the multifidi and has attachments to the IO and TrA; therefore, supplying three-dimensional aid to the lumbar spine and core stability (14). The MRI and ultrasonography imaging showed that during a drawing-in action, the TrA and IO muscles contract bilaterally to form a musculofascial band (like a corset) which improves the stabilization of the lumbopelvic region (15).

A variety of training approaches have been proposed for injury prevention programs including 
neuromuscular control training, core stability training, balance training, and plyometric exercise $(16,17)$. Although the effect of abdominal drawingin maneuver and core muscle activation on some biomechanical parameters has been studied (1118), the effect of doing a few minutes of this maneuver on balance test results has not been established yet. It is hypothesized that better $\operatorname{TrA}$ function during this maneuver can improve balance test results by improving the stabilization of the lumbopelvic region.

\section{Objectives}

This study aimed to determine the effect of exercising a few minutes of ADIM on balance test results.

\section{Methods}

The study participants included 18 apparently healthy participants in the age range of 18-60 years to whom the study objective was explained and written informed consent was obtained from them. It is worth mentioning that, the consent form was prepared according to the Helsinki Declaration for medical research and Shahid Beheshti University of medical sciences ethical guidelines which emphasizes the priority of health and human rights of the participants. Therefore, the exclusion criteria were set accordingly. Participants were excluded if they had unstable conditions (e.g. acute pain in back or knee, moderate/severe dyspnea, abnormal fatigue or muscle weakness), were distressed or agitated, were pregnant, had known cardiovascular disease including hypertension, cancer, diabetes, and pre-diabetes, intermittent claudication, multiple sclerosis (MS), history of vertigo during the previous six months, Parkinson's disease or other neurological conditions which affect the balance control, and major rheumatologic conditions, such as vaculities, rheumatoid arthritis, or lupus.
The height, weight, body mass index (BMI), and abdominal circumference (at the level of anterior superior iliac spines) of participants were measured at the beginning of the test. The participants undertook a fall risk test using the Biodex balance system after proper instruction. In this test, the participants tried to maintain their balance on a fluctuating disc with fixed feet and open eyes. The high fluctuation means poor balance. The test was repeated three times and each time it took about two minutes. The mean scores were measured by the system. Higher scores indicated a poorer balance state. Subsequently, the participants were instructed to perform the abdominal drawing-in maneuver by a verbal cue from the first author. They were asked to gently draw in their navel to their spine while breathing normally. Following the proper instruction of standing abdominal drawingin maneuver, the fall risk test was repeated while the participants were doing this maneuver. The results were compared with initial data obtained from the participants. Data were also analyzed according to BMI (more or less than $25 \mathrm{~kg} / \mathrm{m}^{2}$ ) and abdominal circumference (more or less than 100 $\mathrm{cm}$ ) to analyze the effect of doing ADIM on balance scores in these subgroups. Test results were compared using paired t-test and SPSS software (version 7). A p-value less than 0.05 was considered statistically significant.

\section{Results}

In total, 10 out of 18 participants were male and the rest were female. Descriptive statistics of the participants are presented in Table 1. Totally, significant improvement was observed in the balance score means (lower figures mean better balance scores) after doing abdominal drawing-in maneuver (P-value=0.004). Further analysis revealed a significant improvement (reduction) in the balance score means in individuals with abdominal circumference more than $100 \mathrm{~cm}$ or BMI more than 25 (Table 2).

\begin{tabular}{lcccc}
\hline \multicolumn{1}{l}{ Table 1. General characteristics of the participants. } & & & \\
\hline Variable & Minimum & Maximum & Mean & Standard deviation \\
\hline Age & 20 & 61 & 40.05 & 13.05 \\
Weight & 54 & 92 & 75.00 & 10.67 \\
Height & 146 & 191 & 167.55 & 12.16 \\
Abdominal circumference & 67 & 116 & 98.17 & 10.72 \\
BMI & 18.91 & 31.60 & 26.61 & 3.73 \\
\hline
\end{tabular}

\begin{tabular}{|c|c|c|c|c|c|}
\hline Index & & Number & Mean balance score (before) & Mean balance score (after) & P-value \\
\hline Total participants & & 18 & 2.14 & 1.64 & $0.004^{*}$ \\
\hline \multirow{2}{*}{ BMI } & Less than $25 \mathrm{~kg} / \mathrm{m}^{2}$ & 6 & 1.52 & 1.28 & 0.178 \\
\hline & More than $25 \mathrm{~kg} / \mathrm{m}^{2} \mathrm{~cm}$ & 12 & 2.27 & 1.76 & $0.021 *$ \\
\hline Abdominal & Less than $100 \mathrm{~cm}$ & 10 & 1.59 & 1.38 & 0.086 \\
\hline circumference & More than $100 \mathrm{~cm}$ & 8 & 2.70 & 1.96 & $0.034^{*}$ \\
\hline
\end{tabular}




\section{Discussion}

Based on the obtained results, the performance of the draw-in maneuver improves the results of fall tests. Core muscle training stabilizes the body trunk through the maintenance of an erect posture by resisting the influence of gravity. Core musculature includes abdomen, pelvis, and spine muscles which are involved in the functional movements of extremities (19). Based on the results of a study conducted on healthy subjects, the performance of the abdominal drawing-in maneuver in combination with bridge exercise three times a week for four weeks led to better balance test results (using Tetrax device) compared to when only the bridge exercise is practiced (20). To the best of the authors' knowledge, no other studies evaluated the effect of practicing ADIM on healthy individuals and on the balance test results. In a similar study conducted on healthy subjects, it was revealed that the induction of fatigue separately in trunk extensor and abdominal muscles significantly impaired standing postural control immediately post-fatigue, and the recovery of postural control occurred within two minutes postfatigue. No significant changes in trunk position were observed after recovery and cases could quickly receive somatosensory information. Moreover, the impairment of postural control seemed greater when the trunk extensor muscles rather than the abdominal muscles were fatigued; however, the severity of this impairment was not statistically significant (21). This study highlighted the importance of short-term actions of abdominal muscles in regaining postural control and was unique considering its study design which was somehow similar to that of the present study. Moreover, it supports the idea that the abdominal muscles, such as trunk extensor muscles, have a main short-term role in postural control during upright stance.

In another study conducted on patients with chronic low back pain, it was revealed that doing an abdominal draw-in maneuver three times a week for four weeks increased the TrA muscle thickness (22). In another study performed on stroke patients, the application of four weeks of abdominal drawing-in maneuver, three times per week, 30 minutes each time, resulted in the statistically significant improvement in grip strength, the results of balance test (modified functional reach test) and peak expiratory flow values. The main difference here is that all of the mentioned studies have been done on patients, while this study was conducted on healthy individuals. Moreover, the patients in the other studies had performed abdominal draw-in maneuver weeks before the balance test, while in this study participants learned the maneuver just before the testing.

The improvement of balance test scores following the practice of ADIM in individuals with abdominal obesity and higher BMI in the present study was consistent with findings in other studies which investigated the effect of obesity on postural stability (23-25). Based on the results of these studies, obese individuals have poor postural stability and abdominal obesity is a risk factor for postural instability. In fact, change of body contour induced by doing ADIM can displace the center of gravity backward and reduce anterior pelvic tilt (26) indicating that these individuals need to adopt a less corrective ankle torque for countering gravitational torque.

Regarding the limitations of the present study, one can refer to the fact that instruction of the participants and conduction of the fall risk test before and after instruction was done by the same person, therefore no blinding was performed in this study. Moreover, the performance of correct ADIM maneuver and fall risk test results were influenced by education and training provided to the participants, and since some persons needed more time and training than others to master the maneuver and fall test, this can affect the test results. Therefore, it is suggested that more objective versus subjective balance tests should be adopted in future studies. Finally, the low sample size of the study makes it difficult to generalize the effect of doing ADIM on persons without obesity or abdominal obesity. Accordingly, similar studies should be conducted with larger sample sizes. Furthermore, it is recommended that similar studies be conducted on the effect of ADIM on sportspeople with high mental concentration demands, such as shooting and archery (27).

\section{Conclusion}

The correct performance of ADIM should be educated to people with a high risk of falling, especially in risky situations, such as mass gatherings since a short-term impairment of postural control can be dangerous in these situations, especially in obese elderly individuals. Moreover, ADIM can help sportspeople to improve their sports performance especially in the case of athletes who need high balance demands, such as gymnasts, dancers, and mountain climbers.

\section{Acknowledgments}

The authors would like to thank Dr. Batoul Khoundabi for her help in the analysis of data.

\section{Footnotes}

Author's contributions: Shahram Mohaghegh was responsible for the study conception and design, data collection, and preparing the first draft of the manuscript. Maryam Hajian helped design the study, 
analyzed the data, and supervised the project

Conflicts of Interest: The authors declare that they have no conflict of interest regarding the publication of this study.

\section{Funding/Support: None.}

Ethical considerations: Informed consent was obtained from all participants at the start of the study.

Informed Consent: Informed consent was obtained from all participants at the start of the study.

\section{References}

1. Hrysomallis C. Relationship between balance ability, training and sports injury risk. Sports Med. 2007;37(6):547-56. doi: 10.2165/00007256-200737060-00007. [PubMed: 17503879].

2. Esteghamati A, Khalilzadeh O, Rashidi A, Kamgar M, Meysamie A, Abbasi M. Physical activity in Iran: results of the third national surveillance of risk factors of non-communicable diseases (SuRFNCD-2007). J Phys Act Health. 2011;8(1):27-35. doi: 10.1123/jpah.8.1.27. [PubMed: 21297182].

3. Verhagen E, van der Beek A, Twisk J, Bouter L, Bahr R, van Mechelen W. The effect of a proprioceptive balance board training program for the prevention of ankle sprains: a prospective controlled trial. Am J Sports Med. 2004;32(6):1385-93. doi: 10.1177/0363546503262177. [PubMed: 15310562].

4. Soderman K, Werner S, Pietila T, Engstrom B, Alfredson H. Balance board training: prevention of traumatic injuries of the lower extremities in female soccer players? A prospective randomized intervention study. Knee Surg Sports Traumatol Arthrosc. 2000;8(6):356-63. doi: 10.1007/s001670000147. [PubMed: 11147154].

5. Hodges PW, Richardson CA. Inefficient muscular stabilization of the lumbar spine associated with low back pain. A motor control evaluation of transversus abdominis. Spine. 1996;21(22):2640-50. doi: 10.1097/00007632-19961115000014. [PubMed: 8961451].

6. Hodges PW, Richardson CA. Contraction of the abdominal muscles associated with movement of the lower limb. Phys Ther. 1997;77(2):132-42. doi: 10.1093/ptj/77.2.132. [PubMed: 9037214].

7. Teyhen DS, Miltenberger CE, Deiters HM, Del Toro YM, Pulliam JN, Childs JD, et al. The use of ultrasound imaging of the abdominal drawing-in maneuver in subjects with low back pain. J Orthop Sports Phys Ther. 2005;35(6):346-55. doi: 10.2519/jospt.2005.35.6.346. [PubMed: 16001906].

8. Hodges PW. Changes in motor planning of feedforward postural responses of the trunk muscles in low back pain. Exper Brain Res. 2001;141(2):261-6. doi: 10.1007/s002210100873. [PubMed: 11713638].

9. Akuthota V, Nadler SF. Core strengthening. Arch Phys Med Rehabil. 2004;85(3 Suppl 1):S86-92. doi: 10.1053/j.apmr.2003.12.005. [PubMed: 15034861].

10. Standaert CJ, Herring SA. Expert opinion and controversies in sports and musculoskeletal medicine: the diagnosis and treatment of spondylolysis in adolescent athletes. Arch Phys Med Rehabil. 2007;88(4):537-40. doi: 10.1016/j.apmr.2007.01.007. [PubMed: 17398258].

11. Linde LD, Archibald J, Lampert EC, Srbely JZ. The effect of abdominal muscle activation techniques on trunk and lower limb mechanics during the single-leg squat task in females. $J$ Sport Rehabil. 2018;27(5):438-44. doi: 10.1123/jsr.20160038. [PubMed: 28714764].
12. Ivanenko Y, Gurfinkel VS. Human postural control. Front Neurosci. 2018;12:171. doi: 10.3389/fnins.2018.00171. [PubMed: 29615859].

13. Urquhart DM, Hodges PW, Allen TJ, Story IH. Abdominal muscle recruitment during a range of voluntary exercises. Man Ther. 2005;10(2):144-53. doi: 10.1016/j.math.2004.08.011. [PubMed: 15922235].

14. Richardson CA, Snijders CJ, Hides JA, Damen L, Pas MS, Storm J. The relation between the transversus abdominis muscles, sacroiliac joint mechanics, and low back pain. Spine. 2002;27(4):399-405. doi: 10.1097/00007632-20020215000015. [PubMed: 11840107].

15. Mole JL, Bird M-L, Fell JW. The effect of transversus abdominis activation on exercise-related transient abdominal pain. J Sci Med Sport. 2014;17(3):261-5. doi: 10.1016/j.jsams.2013.05.018. [PubMed: 23849908].

16. Jin Z, Kibler WB, Press J, Sciascia A. The role of core stability in athletic function.J Beijing Sports Univ. 2008;12:039.

17. Hides J, Wilson S, Stanton W, McMahon S, Keto H, McMahon K, et al. An MRI investigation into the function of the transversus abdominis muscle during "drawing-in" of the abdominal wall. Spine. 2006;31(6):E175-8. doi: 10.1097/01.brs.00002027 40.86338.df. [PubMed: 16540858].

18. Jamison ST, McNally MP, Schmitt LC, Chaudhari AM. The effects of core muscle activation on dynamic trunk position and knee abduction moments: implications for ACL injury. J Biomech. 2013;46(13):2236-41. doi: 10.1016/j.jbiomech.2013.06.021. [PubMed: 23891313].

19. Kisner C, Colby L. Resistance exercise. Therapeutic exercise foundations and techniques. $4^{\text {th }}$ ed. Philadelphia: FA Davis; 2002. P. 68-9.

20. Lee JY, Lee DY. The effect of therapeutic abdominal drawing-in maneuver using ultrasonography on lateral abdominal muscle thickness and balance. J Back Musculoskelet Rehabil. 2018;31(6):1139-43. doi: 10.3233/BMR-171080. [PubMed: 30010104].

21. Larson DJ, Brown SH. The effects of trunk extensor and abdominal muscle fatigue on postural control and trunk proprioception in young, healthy individuals. Hum Mov Sci. 2018;57:13-20. doi: 10.1016/j.humov.2017.10.019. [PubMed: 29121507].

22. Park SD, Yu SH. The effects of abdominal draw-in maneuver and core exercise on abdominal muscle thickness and Oswestry disability index in subjects with chronic low back pain. J Exerc Rehabil. 2013;9(2):286-91. doi: 10.12965/jer.130012. [PubMed: 24278873].

23. Son SM. Influence of obesity on postural stability in young adults. Osong Public Health Res Perspect. 2016;7(6):378-81. doi: 10.1016/j.phrp.2016.10.001. [PubMed: 28053843].

24. Cieślińska-Świder J, Furmanek MP, Błaszczyk JW. The influence of adipose tissue location on postural control. J Biomech. 2017;60:162-9. doi: 10.1016/j.jbiomech.2017.06.027. [PubMed: 28705486].

25. Corbeil P, Simoneau M, Rancourt D, Tremblay A, Teasdale N. Increased risk for falling associated with obesity: mathematical modeling of postural control. IEEE Trans Neural Syst Rehabil Eng. 2001;9(2):126-36. doi: 10.1109/7333.928572. [PubMed: 11474965].

26. Stefani L, Galanti G, Padulo J, Bragazzi NL, Maffulli N. Sexual activity before sports competition: a systematic review. Front Physiol. 2016;7:246. doi: 10.3389/fphys.2016.00246. [PubMed: 27445838].

27. Soori M, Mohaghegh S, Hajian M, Abedi Yekta A. Sexual activity before competition and athletic performance: A systematic review. Ann Appl Sport Sci. 2017;5(3):5-12. doi: 10.29252/acadpub.aassjournal.5.3.5. 